\title{
Indicadores de desempenho para bibliotecas universitárias: definições e aplicações sob o ponto de vista da literatura
}

Teresinha das Graças Coletta
Mestranda em engenharia de produção e Diretora do Serviço de Biblioteca da EESC/USP

Henrique Rozenfeld

Prof. do Departamento de Engenharia de Produção - EESC/USP

O artigo apresenta discussões da literatura sobre indicadores de desempenho para bibliotecas universitárias. Apresenta ainda um breve relato sobre a importância da avaliação e medição de desempenho nas organizações em geral e, em especial, nas bibliotecas universitárias. Demonstra que uma maior preocupação é observada na literatura internacional e que há falta de definição e uso de indicadores no Brasil, onde a literatura é mais restrita. Questiona sobre o uso de metodologia única de avaliação para as bibliotecas e sugere $o$ aprofundamento dos estudos e a conscientização sobre a importância do uso de indicadores de desempenho nas bibliotecas universitárias brasileiras.

Palavras-Chave: Indicadores de desempenho. Bibliotecas universitárias; Avaliação de desempenho.

\section{Performance indicators for university libraries: definitions and applications from the point of view of the literature.}

The article presents discussions on the literature about performance indicators for university libraries. It presents a concise report on the importance of evaluation procedures and performance measurements in organizations and libraries in general, especially university libraries. It is demonstrated that there is a great international but not Brazilian literature production on the 
topic. The use of a unique evaluation methodology for all libraries was questioned. Further studies on this subject were suggested and the need of performance indicators for Brazilian university libraries was emphasized.

Keywords: Performance indicators. University libraries; Performance evaluation.

Recebido em 04 12.2006 Aceito em 01.10.2007

\section{Introdução}

A qualidade nas organizações tem sido tratada como forma de sobrevivência no mercado cada vez mais competitivo. Isso também pode ser aplicado às organizações não lucrativas, em especial às bibliotecas universitárias públicas, que dão suporte ao ensino e à pesquisa que as instituições oferecem tanto em nível de graduação quanto de pósgraduação.

Para medir a qualidade é preciso avaliar, e segundo Lancaster (1996, p.1), "uma avaliação é feita não como um exercício intelectual, mas para reunir dados úteis para atividades destinadas a solucionar problemas ou tomar decisões". E ao considerar a biblioteca como "uma interface entre os recursos de informação disponíveis e a comunidade de usuários a ser servida", o autor ressalta que qualquer avaliação deve medir o êxito dessa interface.

Nesse contexto, vale lembrar as cinco Leis de Ranganathan (1963): "os livros são para usar"; "a cada leitor seu livro"; "a cada livro seu leitor"; "poupe o tempo do leitor" e "a biblioteca é um organismo em crescimento". Embora tenham sido estabelecidas em 1931, elas continuam válidas e deveriam ser o parâmetro inicial para qualquer avaliação e, a partir disso, para a medição de desempenho.

Recomenda-se o uso de indicadores para medir o desempenho das organizações e, segundo a ISO (1998, p. 02 ), indicador é:

Uma expressão (que pode ser numérica, simbólica ou verbal) usada para caracterizar atividades (eventos, objetos, pessoas) em termos quantitativos e qualitativos, a fim de determinar o valor das atividades caracterizadas e os métodos associados.

Trzesniak (1998) faz uma reflexão sobre as virtudes e as limitações dos indicadores; trata da sua construção e divide esse processo em três fases:

1. a fase que precede a obtenção da informação, que inclui a proposição e a padronização da metodologia;

2. a fase de obtenção da informação, que envolve a reelaboração e a interpretação desses dados;

3. a fase de aperfeiçoamento da relação indicador - informação, que trata do refinamento e da identificação dos valores de referência. 
Ele ressalta que qualquer indicador deve ter, a priori, as seguintes características: relevância; graduação de intensidade; univocidade; padronização; rastreabilidade. E indica as características desejáveis: cobertura (ou amplitude ou abrangência); portabilidade (ou transferabilidade) e invariância de escala. Mesmo com estas características, enfatiza que um indicador pode ser excelente em um determinado contexto, mas não recomendado e até mesmo não aplicável em outro. Ou seja: "A portabilidade não é obvia, não é evidente, não é dada a priori. Precisa ser verificada e só deve ser aceita quando exaustivamente comprovada" (TRZESNIAK, 1998, p.163).

"Disponibilizar indicadores é uma enorme responsabilidade" e não é nada "trivial, mas pode e deve ser feito" (TRZESNIAK, 1998, p. 164). Para o autor, a disponibilidade de computadores, além de reduzir drasticamente o custo do armazenamento e manipulação de grandes massas de dados, permite a padronização de procedimentos com menor esforço. Isso viabiliza a obtenção de indicadores variados, disponíveis nos próprios sistemas que guardam e disseminam informações em geral. O autor alerta para a importância de se extrair dos indicadores as informações para as quais eles foram projetados, caso contrário os dados não terão significado. Além disso, lembra a necessidade de revisão constante e da divulgação clara para todos os interessados.

Gil (1992) destaca que um indicador pode ser considerado um "termômetro" para a alta administração, com capacidade para identificar, externamente à organização, o comportamento dos clientes. Destaca ainda que o indicador tem um ciclo de vida, e isso deve ser considerado para que reflita a realidade organizacional.

\section{Sistemas de medição de desempenho nas organizações (MD)}

Para Kiyan (2001) a MD é um conceito multidisciplinar e sua operacionalização se dá através de indicadores, que buscam quantificar o desempenho do objeto de estudo. A MD pode ser empregada tanto no âmbito interno quanto externo das organizações. Um ponto importante são as características dos indicadores, apresentadas no quadro a seguir (QUADRO 1):

QUADRO 1- Características dos indicadores

\begin{tabular}{l|l|l}
\hline \multicolumn{1}{c|}{ Aspectos } & \multicolumn{1}{c}{ Curto prazo } & \multicolumn{1}{c}{ Longo prazo } \\
\hline Foco & Operacional & Estratégico \\
\hline Orientada & A ação & A análise \\
\hline Freqüência & Contínua & Esparsas \\
\hline Nível de agregação & Pequeno & Grande \\
\hline Concentração de dados & Maior parte no processo & Em vários processos \\
\hline Papel & Controlar & Explorar \\
\hline Natureza & Maior parte não financeira & Maior parte financeira \\
\hline FONTE - Adaptado de KIYAN (2001). &
\end{tabular}


Quando se trata da avaliação do desempenho empresarial no âmbito de um modelo de gestão, e a partir dos processos da organização, é possível definir os indicadores que Rodriguez y Rodriguez (2001) classificam em:indicadores de resultados: são aqueles relacionados aos objetivos. São definidos a partir do desdobramento da visão. Parte-se da definição de macroindicadores que devem ser desdobrados desde o nível estratégico até o operacional;

indicadores críticos: são indicadores relacionados às estratégias, ou seja, aos processos críticos;

indicadores de desempenho: os indicadores que estão relacionados aos demais processos de negócio.

Ao se definir indicadores, independente da visão de cada responsável por um determinado conjunto, é necessário julgar quais são efetivamente mais importantes para a organização. Recomenda-se a definição de um conjunto pequeno e balanceado de indicadores, pois em grande quantidade estes podem dificultar e gerar a perda de foco da alta administração. É razoável ter-se até dez, os quais podem ser desdobrados para gerar outros, até centenas deles, se necessários.

A MD nos moldes tradicionais era fortemente orientada pela visão financeira. Mais recentemente, Ghalayini e Noble (1996) tratam das mudanças ocorridas, e apresentam as características dos sistemas de medição não-tradicionais que buscam: a) medidas relacionadas à estratégia de manufatura; b) medidas não financeiras (de uso restrito aos gerentes); c) informações de interesse dos supervisores e operadores para uso em decisões diárias; d) medidas simples para que os operadores do chão de fábrica possam usá-las e entendê-las facilmente; e) medidas que podem promover a melhoria em vez de só controlar; f) medidas que devem mudar de acordo com a dinâmica do mercado. Nesse contexto, apresentam alguns sistemas integrados de MD mais comumente utilizados:

- SMART System - Strategic Measurements, Analysis and Reporting Technique: a vantagem é integrar os objetivos corporativos com os indicadores operacionais;

- PMQ - Performance Measurement Questionnaire: ajuda os gerentes a identificar as necessidades de melhoria; determinar a extensão das medidas de desempenho que sustentam as melhorias; estabelecer uma agenda para melhorias de medidas de desempenho;

- BSc - Balanced Scorecard: é um sistema de gerenciamento estratégico complementar ao controle financeiro tradicional, que monitora as estratégias de longo prazo por meio de mecanismos de mensuração. Mede essencialmente: perspectiva do cliente; perspectiva interna; inovação e aprendizagem; perspectiva financeira.

Mesmo com características mais complexas, os sistemas integrados de medição de desempenho apresentam limitações. São ferramentas mais de controle do que de melhoria; não definem qual objetivo pode ser alcançado em um horizonte específico de tempo; não são sistemas 
dinâmicos; referem-se somente ao desempenho do momento; a maioria não ressalta a importância do tempo como estratégia para medida de desempenho.

Bourne e Neely (2002), ao apresentarem o resultado de uma pesquisa com altos gerentes sobre 0 sucesso $e$ as falhas na implementação de medidas de desempenho em dez empresas, tratam de:

- Razões para continuar: os benefícios das medições, o comprometimento contínuo da alta gerência, etc.

- Algumas dificuldades para continuar: dificuldades de acesso aos dados e sistemas de tecnologia de informação, tempo e esforço requeridos, etc.

- Algumas razões para não implementar: tempo e esforço requeridos, falta de benefícios resultantes das medições, comprometimento contínuo da alta gerência, etc.

É importante lembrar que a implementação de sistemas de medidas, em qualquer tipo de organização, requer o comprometimento da alta gerência. Mas, acima de tudo, é necessário que a organização tenha certeza de seu propósito, bem como esteja estruturalmente preparada e que tenha uma cultura voltada para isso. $E$ nas organizações não lucrativas, como é o caso das bibliotecas, maior deve ser o comprometimento da alta administração.

\section{A medição de desempenho em bibliotecas universitárias}

Quando se trata do assunto no Brasil, pouco se tem de prática para exemplificar. Não há ações nacionais para discutir o assunto, ou seja, não há um plano nacional de avaliação da qualidade nas universidades, como há na Europa (ALONSO ARÉVALO et al., 1999); para a avaliação do ensino, da pesquisa e dos serviços e, nesse contexto, a avaliação das bibliotecas como suporte aos três pilares institucionais.

$\mathrm{Na}$ literatura internacional, Evans et al. (1972) identificam critérios básicos para definir medidas de avaliação em bibliotecas, a saber: acessibilidade; custos; satisfação do usuário; tempo de resposta do sistema; proporção custo/benefício; uso.

Por outro lado, Lancaster (1980, apud LUZ, 1989) questiona a abrangência desses critérios e propõe: cobertura da coleção; recuperação; precisão; esforço despendido pelo usuário; tempo de resposta do sistema; forma de saída (output) proporcionada pelo sistema. E na Europa, Moore (1989, apud ROSADOS, 2005), dentre vários pontos relevantes em seu trabalho, trata da necessidade de adequação dos critérios para uso de cada biblioteca, pois é praticamente impossível existir um manual para aplicação geral.

Luz (1989), ao propor um modelo de avaliação para bibliotecas universitárias no Brasil, menciona a necessidade da definição de indicadores de desempenho. O modelo proposto por Luz não deixa claro os indicadores para análise, mas se baseia em três critérios de 
desempenho: opinião dos usuários; utilização das coleções; disponibilidade das coleções. Vale lembrar que a primeira menção à necessidade de indicadores no país surgiu como recomendação do $2^{\circ}$. SNBU - Seminário Nacional de Bibliotecas Universitárias, em 1981.

Em um trabalho mais abrangente, Macedo e Dias (1992) descrevem a biblioteca universitária dentro dos parâmetros de tipologia, propósitos, objetivos, organização e administração, serviços, materiais e agentes. Elas não estendem o trabalho ao âmbito qualitativo, mas fornecem subsídios para a caracterização da biblioteca universitária, o que até então não havia sido devidamente sistematizado na literatura.

Em uma tentativa de melhor tratar das questões relativas aos padrões para bibliotecas universitárias no Brasil, Carvalho (1995) enfatiza sua importância como mecanismo de suporte à avaliação organizacional. Para embasar seu trabalho, apresenta os dez padrões da IFLA (STANDARDS..., 1987), como orientação geral; além de ressaltar a importância de se definir padrões nacionais, regionais e locais para as bibliotecas universitárias.

Sumsion e Ward (1995), ao considerarem os indicadores de desempenho em um contexto amplo, entendem que seus propósitos são: monitorar o desempenho; monitorar os resultados da inovação; identificar problemas e oportunidades; avaliar as opções alternativas; planejar. Eles ressaltam que muitos indicadores podem ser utilizados para todos os tipos de bibliotecas, mas nesse estudo feito na Europa, atenção especial foi dada às bibliotecas universitárias e públicas.

Segundo a ISO (1998, p. 04),

O propósito dos indicadores de desempenho é funcionar como uma ferramenta para determinar a qualidade e a eficácia dos serviços oferecidos pela biblioteca e outras atividades relacionadas à biblioteca e para determinar a eficiência dos recursos alocados pela biblioteca para cada serviço e outras atividades.

E para medir e examinar a qualidade de um indicador de desempenho, é recomendado pela ISO (1998, p. 04-05), o uso de 6 (seis) critérios:

- Conteúdo informativo: deve servir de ferramenta para identificar sucessos, problemas e falhas no desempenho da biblioteca. Deve fornecer informações para a tomada de decisão;

- Confiabilidade: deve produzir os mesmos resultados quando usado repetidamente nas mesmas circunstâncias;

- Validade: o indicador deve ser válido, ou seja, deve medir o que se pretende medir;

- Pertinência: o indicador deve ser apropriado ao propósito para o qual foi estabelecido.

- Praticidade: o indicador deve ser prático. Deve usar os dados disponíveis na biblioteca, despendendo um mínimo de tempo e 
qualificação do pessoal, custo operacional e tempo dos usuários;

- Comparabilidade: o indicador de desempenho permite a comparação entre bibliotecas similares, ou seja, que tenham o mesmo nível de qualidade ou o mesmo nível de eficiência.

A partir de uma lista de 32 (trinta e dois) indicadores utilizados nas bibliotecas em geral, cada biblioteca deve decidir quais são representativos para o seu tipo em particular e para o que ela deseja medir (ISO, 1998).

Quanto às razões possíveis para se avaliar os serviços de uma biblioteca, Lancaster (1996) elenca:

- estabelecer uma "escala" para mostrar qual o nível de desempenho da biblioteca no momento;

- comparar o desempenho de uma biblioteca com outras;

- justificar a sua existência;

- identificar as possíveis causas de fracasso ou ineficiência do serviço para melhorá-lo.

A "avaliação não é um fim em si mesma" e deveria ser realizada somente em função de objetivos específicos e para melhorar o que já foi feito, ou seja, o estudo deve ser.

planejado para responder certas questões específicas e para reunir dados que permitam melhorar, [pois o] [...] Investimento num estudo avaliatório criterioso talvez se justifique inteiramente, se os resultados revelarem o que deve ser feito para melhorar a eficácia ou a relação custo-eficácia do serviço ou sua relevância para as necessidades atuais da comunidade (LANCASTER, 1996, p.15-16).

Segundo Ponjuan Dante (1998), a "aplicação está associada à identificação de parâmetros de eficácia dos serviços oferecidos tanto como para monitorar o andamento das atividades desenvolvidas e o rendimento do pessoal". E reforçando outros autores, Ponjuan (1998, p. 35) afirma:

Os indicadores são ferramentas de gestão que permitem precisar a qualidade e eficácia dos serviços oferecidos e de outras atividades desenvolvidas em diversas organizações. [...] os indicadores estão muito vinculados aos objetivos da organização e constituem ferramentas imprescindíveis para o planejamento e avaliação.

Para Cullen (1998), existem duas razões que afetam o uso de sistemas de avaliação de desempenho nas bibliotecas:

1. não há incentivo forte o suficiente: a administração de serviços e de informação não são casos de vida ou morte, não 
têm de gerar lucro, nem satisfazer os provedores e raramente têm de comprovar seu valor;

2. para medir o desempenho de bibliotecas/serviços de informação, se lida com resultados / impactos imprecisos. Por exemplo, não há uma definição clara dos impactos sociais, não há também uma definição do custo/beneficio do serviço de informação para a organização. Por ser uma área que envolve o comportamento humano, não há benefícios específicos, como no caso da medicina, onde se pode medir o valor do trabalho ou o impacto negativo gerado.

Stubbs (2004) trata da natureza, da utilidade e da construção de indicadores de desempenho, e adota a definição da ISO. Através do estudo de vários autores, fica clara para ele a diferença entre estatística e avaliação. Todo processo de avaliação reflete um juízo de valor, enquanto que as estatísticas são dados puros, coletados automaticamente. Também nessa linha das diferenças entre ambas, a IFLA (1998 apud STUBBS, 2004, p.150) lembra que "as estatísticas tratam dos dados de quem usa a biblioteca, mas não de quem não usa; as estatísticas arrolam dados exatos, fáceis de coletar". A avaliação de desempenho, por outro lado, compara os dados e os combina entre si, e leva em conta a avaliação dos usuários, além de comparar os dados com as metas da biblioteca. Para o autor:

Uma biblioteca tem várias razões para medir seu desempenho; por razões políticas, incluindo compromissos vinculados com as políticas nacionais de informação de um país, responsabilidade ante aos que apóiam política e financeiramente o funcionamento e o desenvolvimento da biblioteca, para mostrar os resultados dos sucessos e melhorias obtidas aos usuários e à comunidade em geral, como auxílio na tomada de decisão e como ferramenta de gestão (STUBBS, 2004, p.151).

Ao tratar de um Modelo de Gestão proposto para o SIBi/USP (Sistema Integrado de Bibliotecas da Universidade de São Paulo), Coletta et. al. (2002 e 2004) apresentam um mapeamento inicial de processos e indicadores de desempenho (Quadro 2). As autoras sugerem que 0 desdobramento desses indicadores seja feito de acordo com metodologias já consolidadas na literatura como, por exemplo, a da ISO (1998) ${ }^{1}$.

\footnotetext{
1 Os processos em destaque foram definidos na segunda etapa do projeto, quando não se trabalhou com indicadores.
} 
QUADRO 2 - Processos de trabalho e indicadores de desempenho

\begin{tabular}{|c|c|}
\hline Processo & Indicador \\
\hline Planejamento estratégico & $\begin{array}{l}\text { a) atualização constante de missão e visão } \\
\text { b) cumprimento das metas estabelecidas }\end{array}$ \\
\hline Marketing de produtos e serviços & $\begin{array}{l}\text { - } \text { número de usuários dos produtos / serviços } \\
\text { - } \quad \text { visibilidade dos produtos / serviços } \\
\text { - } \quad \text { aumento do valor da imagem da organização }\end{array}$ \\
\hline 1.1.1.1 Gestão de projetos & - $\quad$ indicadores não definidos * \\
\hline 1.1.1.2 Gestão do conbecimento & - indicadores não definidos * \\
\hline $\begin{array}{l}\text { Formação e desenvolvimento do } \\
\text { acervo }\end{array}$ & $\begin{array}{l}\text { - } \quad \text { uso da coleção } \\
\text { - } \text { disponibilidade de títulos e exemplares } \\
\text { - } \text { tempo médio de aquisição } \\
\text { - número de licenças para acesso eletrônico } \\
\text { - critérios para seleção, aquisição e avaliação } \\
\text { - contínua } \\
\text { - benchmarking }\end{array}$ \\
\hline Tratamento da informação & $\begin{array}{l}\text { 1. custo médio da catalogação por título } \\
\text { 2. tempo médio de processamento do documento } \\
\text { 3. organização de catálogos de acordo com } \\
\text { padronização internacional }\end{array}$ \\
\hline $\begin{array}{l}\text { Mediação do acesso à } \\
\text { informação }\end{array}$ & $\begin{array}{l}\text { - } \text { satisfação do cliente } \\
\text { - } \quad \text { rapidez e eficiência na provisão de EEB } \\
\text { - } \quad \text { tempo de acesso compatível com as } \\
\text { necessidades dos clientes } \\
\text { - } \quad \text { promoção, manutenção e avaliação da } \\
\text { qualidade dos produtos / serviços } \\
\text { - facilidade de acesso ao documento local e } \\
\text { remoto } \\
\text { - } \begin{array}{l}\text { oferecimento de oportunidades de educação } \\
\text { formal e informal aos clientes }\end{array}\end{array}$ \\
\hline Gerenciamento da infra-estrutura & - indicadores não definidos * \\
\hline $\begin{array}{l}\text { Gerenciamento de recursos } \\
\text { financeiros }\end{array}$ & $\begin{array}{l}\text { - } \quad \text { administração de acordo com os objetivos } \\
\text { - } \quad \text { uso do recursos com eficiência e eficácia }\end{array}$ \\
\hline Gerenciamento de pessoas & $\begin{array}{l}\text { - } \text { adequação do número e da qualificação } \\
\text { pessoal } \\
\text { - } \text { apoio financeiro para garantia da qualificação } \\
\text { contínua } \\
\text { - satisfação e comprometimento da equipe }\end{array}$ \\
\hline
\end{tabular}

FONTE - COLETTA et. al. (2002, 2004)

Outro trabalho de identificação e avaliação dos principais indicadores para gestão estratégica foi feito por Martins (2004, p. 94), no Sistema de Bibliotecas da Unicamp (SBU), e destaca a ausência de "sistemas de medição gerenciais adequados e do registro sistemático de dados" que viabilizariam a participação coletiva no planejamento e gestão das organizações. 


\section{Discussão}

Um ponto chave para as bibliotecas universitárias brasileiras é a discussão sobre a necessidade ou importância da avaliação. A pergunta é: por que avaliar? Embora já haja algum tipo de cobrança sobre seu desempenho, possivelmente influenciada pelos investimentos feitos pelas próprias universidades e pelo Governo Federal através da Capes (Ex. Portal de Periódicos), aliada à criação e disseminação dos prêmios de qualidade em nível estadual e nacional, pouco se vê de realização prática.

A literatura confirma as razões disso, como apontam Lancaster (1996), Cullen (1998) e Stubbs (2004). Dentre as várias razões que indica, Lancaster (1996) cita a necessidade de justificar a sua existência e de comparar o desempenho de uma biblioteca com outras. Mas não é comum no Brasil esse tipo de procedimento, mesmo em sistemas de bibliotecas onde, através de relatórios e dados estatísticos, são feitos alguns mapeamentos, embora sem um cunho comparativo para, por exemplo, a distribuição de recursos.

Cullen (1998) é um pouco mais drástico ao afirmar, como dissemos, que, via de regra, as bibliotecas não têm incentivo forte o suficiente para realizar avaliações, pois não são casos de vida ou morte, não tem de gerar lucro, nem satisfazer os provedores e raramente têm de comprovar seu valor. Também diz que, no caso das bibliotecas, os resultados/impactos são imprecisos, e compara com a medicina, onde se pode medir o valor do trabalho ou o impacto negativo gerado. Isso faz sentido, pois os impactos sociais são percebidos mais em longo prazo e suas causas são mais difíceis de precisar.

Por outro lado, Stubbs (2004), numa visão mais recente, é mais brando. Diz que uma biblioteca tem razões políticas frente aos que a apóiam, e deve mostrar os resultados dos sucessos e melhorias obtidos aos usuários e à comunidade em geral. Os dados de avaliação servem ainda como auxílio à tomada de decisão e também como ferramenta de gestão.

Outro ponto que merece destaque é a dificuldade de se definir indicadores (EVANS, 1972; LANCASTER, 1996 e ISO, 1998) e de se criar um manual de avaliação facilmente aplicável a qualquer biblioteca, como destacam Moore (1989) e Rosados (2005).

Diante dessa discussão, como contrapartida ao investimento da Capes com seu Portal de Periódicos e em resposta a estudos como o de Martins (2004), pergunta-se: as bibliotecas beneficiárias deveriam ser as bibliotecas beneficiárias ser avaliadas com base nos mesmos indicadores? Quais instituições estão devidamente credenciadas para conduzir um processo de avaliação dessa natureza?

\section{Considerações finais}

Tendo em vista a literatura apresentada, sugere-se uma investigação junto às bibliotecas universitárias brasileiras para elaborar um diagnóstico da prática de avaliação de desempenho, com especial 
atenção à definição e uso de indicadores. Justifica-se essa necessidade cada vez mais, em especial para demonstrar o potencial e a qualidade do acervo, da gestão e dos serviços prestados pelas bibliotecas enquanto suporte do ensino, da pesquisa e extensão nas universidades.

Quando se trata da avaliação dos cursos de graduação e pósgraduação, a biblioteca, enquanto infra-estrutura de funcionamento, também passa por avaliações. Embora estas, na maioria das vezes, se restrinjam a breves visitas, cujos resultados dependem do olhar de cada avaliador. Não existe um planejamento para a elaboração dessas avaliações. Não há, nas bibliotecas, dados comparativos com outras da mesma área que demonstrem um melhor ou pior desempenho.

Deveria haver, também, por parte da alta administração, em especial dos órgãos públicos como a Capes, uma cobrança nesse sentido. E o IBICT (Instituto Brasileiro de Informação em Ciência e Tecnologia) poderia tomar a frente de um estudo nacional para o estabelecimento de metodologias de avaliação baseadas em indicadores de desempenho para as bibliotecas universitárias.

Vale lembrar que existem, no país, algumas bibliotecas participando dos prêmios estaduais e nacionais de qualidade, que tomam por base toda a infra-estrutura do PNQ (Prêmio Nacional da Qualidade) e sob a liderança da administração local. São exemplos disso as Biblioteca da FGV-Rio (Fundação Getúlio Vargas) e da ESALQ-USP (Escola Superior de Agricultura "Luiz de Queiroz").

\section{Referências}

ALONSO ARÉVALO, J. et al. La gestión de las bibliotecas universitarias: indicadores para su evaluación. In: SEMINARIO INDICADORES EN LA UNIVERSIDAD, León, 9 - 11 de junio de 1999. Información y decisiones. Léon: Universidad de León, 1999. Disponível em: $<$ http://eprints.rclis.org/archive/00000492/01/Indicadores.pdf>. Acesso em: 18 jun. 2005.

BOURNE, M.; NEELY, A. The success and failure of performance measurement initiatives: perceptions of participating managers. International Journal of Operations \& Production Management, v.22, n.11, p.1288-1310, 2002.

CARVALHO, M. C. R. Estatísticas e padrões para o planejamento e a avaliação de bibliotecas universitárias. Florianópolis: UFSC, 1995.

COLETTA, T. G. et al. Modelo de gestão: proposta para o SIBi/USP. In: Seminário Nacional de Bibliotecas Universitárias, 12., Recife, 2002. Anais... Recife, UFPb, 2002. Cd. Rom.

. et al. Modelo de gestão: proposta para o SIBi/USP. 2004. Disponível em: <http://www.sibi.usp.br/gestao>. Acesso em: 10 jun. 2005. Documentação final de projeto.

CULLEN, R. Measure for measure: a post modern critique of performance measurement in libraries and information services. In: Iatul Conference 
Proceedings, 1st June - 5th June, 1998, University of Pretoria, South Africa. Disponível

em: <http://www.iatul.org/conference/proceedings/vol08/papers/cullen.html > .

Acesso em: 01 jul. 2005.

EVANS, E. et al. Review of criteria used to measure library effectiveness. Bulletin of the Medical Library Association, v. 60, n. 1, p.102-10, Jan. 1972.

GHALAYINI, A.M.; NOBLE, J.S. The changing basis of performance measurement. International Journal of Operations \& Production Management, v.16, n.8, p.63-80, 1996.

GIL, A. L. Qualidade total nas organizações: indicadores de qualidade, gestão de qualidade. São Paulo: Atlas, 1992.

INTERNATIONAL FEDERATION OF LIBRARIES ASSOCIATIONS - IFLA. Medicion de la calidad: directrices internacionales para la medición del rendimiento de las bibliotecas universitarias. Madrid: ANABAD, 1998. Apud Stubbs, 2004, p. 150 apud STUBBS, E. A. Indicadores de desempeño: naturaleza, utilidad y construcción. Ciência da Informação, v.33, n.1, p.149-154, abr. 2004.

INTERNATIONAL ORGANIZATION FOR STANDADIZATION - ISO. ISO 11620: Information and documentation - library performance indicators. Genève, 1998. 56p.

KIYAN, F. M. Proposta para desenvolvimento de indicadores de desempenho como suporte estratégico. 2001. Dissertação (Mestrado) Escola de Engenharia de São Carlos, Universidade de São Paulo, São Carlos, 2001.

LANCASTER, F.W. Avaliação de serviços de bibliotecas. Brasília: Briquet de Lemos, 1996.

. The measurement and evaluation of library services. Arlington, Va: Information Research Center, 1980. Apud Luz, p.26 apud LUZ, G. M. S. Bibliotecas universitárias: um modelo de avaliação de desempenho. 1989. Tese (Doutorado) - Escola de Comunicações e Artes da Universidade de São Paulo, São Paulo, 1989.

LUZ, G. M. S. Bibliotecas universitárias: um modelo de avaliação de desempenho. 1989. Tese (Doutorado) - Escola de Comunicações e Artes da Universidade de São Paulo, São Paulo, 1989.

MACEDO, N. D.; DIAS, M. M. K. Subsídios para a caracterização da biblioteca universitária. Revista Brasileira de Biblioteconomia e Documentação, v.25, n. 3/4, p. 40-48, jul./dez. 1992.

MARTINS, V. S. G. Sistemas de Informação das Bibliotecas da UNICAMP: identificação e avaliação dos principais indicadores para gestão estratégica. 2004. Dissertação (Mestrado Profissional) - Universidade Estadual de Campinas, Campinas, 2004. 
MOORE, N. Comment mensurer I'efficacité des bibliotèques publiques: project de manuel. Paris: UNESCO, 1989. Apud Rosados, p.70 apud ROSADOS, H.B.F.R. Uso de indicadores na gestão de recursos de informação. Revista Digital de Biblioteconomia e Ciência da Informação, Campinas, v.3, n.1, p.60-76, jul./dez.2005. Disponível em: $<$ http://server01.bc.unicamp.br/seer/ojs/index.php>. Acesso em: 10 mar. 2007.

PONJUÁN DANTE, G. Gestión de información en las organizaciones: principios, conceptos y aplicaciones. Santiago de Chile: CECAPI, 1998.

RANGANATHAN, S. R. The five laws of library science. Bombay: Asia Publ. House, 1963.

RODRIGUES y RODRIGUES, M. V. Gestão do conhecimento: reinventando a empresa para uma sociedade baseada em valores intangíveis. Rio de Janeiro: IBPI Press, 2001.

ROSADOS, H. B. F. R. Uso de indicadores na gestão de recursos de informação. Revista Digital de Biblioteconomia e Ciência da Informação, Campinas, v.3, n.1, p.60-76, jul./dez.2005. Disponível em: $<$ http://server01.bc.unicamp.br/seer/ojs/index.php>. Acesso em: 10 mar. 2007

Standards for university libraries. IFLA Journal, v.13, n.2, p.120-125, 1987.

STUBBS, E. A. Indicadores de desempeño: naturaleza, utilidad y construcción. Ciência da Informação, v.33, n.1, p.149-154, abr. 2004.

SUMSION, J.; WARD, S. The next generation of performance indicators. Disponível em: <http://www.ifla.org//V/ifla61/61-sumj.htm>. Acesso em: 18 jun. 2005.

TRZESNIAK, P. Indicadores quantitativos: reflexões que antecedem seu estabelecimento. Ciência da Informação, v.27, n.2, p.159-164, maio/ago. 1998. 